\title{
Changes in Healthcare Resource Use and Costs in Commercially Insured Insomnia Patients Initiating Suvorexant
}

\author{
Hrishikesh P. Kale - Zaina P. Qureshi (D) - Ruchit Shah • \\ Rezaul Khandker · Marc Botteman · Weilin Meng · Ruth Benca
}

Received: June 30, 2021 / Accepted: August 6, 2021

(C) The Author(s) 2021

\begin{abstract}
Introduction: Insomnia diagnosis has been associated with a significant clinical and economic burden on patients and healthcare systems. This study examined changes in healthcare resource use (HCRU) and costs in insomnia patients before and after initiation of suvorexant treatment.

Methods: This retrospective cohort study analyzed Optum Clinformatics Data Mart claims data (Jan 2010-Dec 2018). Patients with $\geq 2$ insomnia diagnosis claims and $\geq 1$ prescription for suvorexant were included. Prevalent and incident insomnia patients were analyzed separately. The change in the trends of HCRU and costs were examined for 12 months before and 12 months after suvorexant initiation. An interrupted time series (ITS) analysis was
\end{abstract}

Supplementary Information The online version contains supplementary material available at https:// doi.org/10.1007/s12325-021-01891-8.

H. P. Kale $\cdot$ R. Shah $\cdot$ M. Botteman

OPEN Health Evidence \& Access, Bethesda, MD, USA

Z. P. Qureshi $(\varangle) \cdot$ R. Khandker · W. Meng

Merck \& Co., Inc, 2000 Galloping Hill Rd,

Kenilworth, NJ, USA

e-mail: zaina.qureshi@merck.com

R. Benca

University of California, Irvine, Irvine, CA, USA conducted to assess the level and slope changes. Subgroups of patients with mental health comorbidities were examined.

Results: The study included 18,919 and 5939 patients in the prevalent and incident insomnia cohorts, respectively. For the prevalent cohort, mean (SD) age was 64.5 (14.1) years, 65\% were female, $74 \%$ had Medicare Advantage coverage, and $61 \%$ had a Charlson comorbidity index score $\geq 1$. Characteristics for the incident cohort were similar. The ITS results suggested that the trend for monthly total healthcare cost (THC) was increasing before suvorexant initiation (US\$52.51 in the prevalent cohort, $\$ 74.93$ in incident insomnia cohort), but, after suvorexant initiation, the monthly total cost showed a decreasing trend in both cohorts. The decrease in slope for THC after suvorexant initiation were $\$ 72.66$ and $\$ 112.07$ per month in the prevalent and incident cohorts, respectively. The monthly trends in HCRU rates also decreased. The subgroup analysis showed that decreases were 1.5-3 times greater for patients with mental health comorbidities.

Conclusions: In this real-world study, suvorexant initiation was associated with immediate and continued decreases in HCRU and costs in insomnia patients. Further research is needed to understand the effect of suvorexant initiation on direct medical costs as well as costs associated with lost productivity in other real-world settings. 


\section{Graphical Abstract:}

CHANGES IN HEALTHCARE RESOURCE USE (HCRU) AND COSTS IN COMMERCIALLY INSURED INSOMNIA PATIENTS INITIATING SUVOREXANT

Hrishikesh P. Kale; Zaina P. Qureshi; Ruchit Shah; Rezaul Khandker; Marc Botteman; Weilin Meng; Ruth Benca DOI: https://doi.org/10.1007/s12325-021-01891-8

SUVOREXANT WAS ASSOCIATED WITH A DECREASE IN ALL-CAUSE HCRU AND COSTS IN A 1-YEAR REAL-WORLD STUDY

Burden of Insomnia Disorder in the US

Prevalence
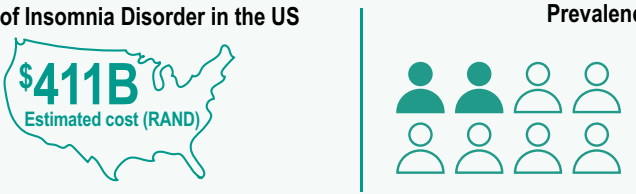

$10-25 \%$

but may vary from $5 \%$ to $50 \%$

PREVALENT COHORT

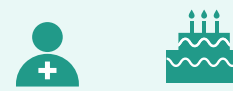

$18,91964.5(14.1)$

Patients Mean (SD) Age

(Years)

1

$65 \%$

Female

\section{$74 \% / 26 \%$}

Medicare/Commercial

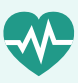

$68 \%$
MENTAL HEALTH COMORBIDITIES

Among a subgroup of patients with mental health disorders on suvorexant, cost reductions were 1.5-3 times greater than those for the overall cohort.

ANALYSIS

Interrupted Time Series (ITS)

Level and slope changes
INCIDENT COHORT
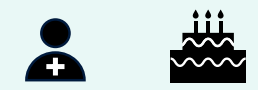

$5,939 \quad 62.8(15.5)$

Patients Mean (SD) Age

(Years)

Y

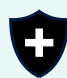

$63 \% \quad 71 \% / 29 \%$

Female Medicare/Commercial

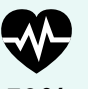

$59 \%$

$\mathrm{CCl}$ index score $\geq 1$

$\mathrm{CCl}$ index score $\geq 1$

$\$ 4,009$

$\$ 3,848$

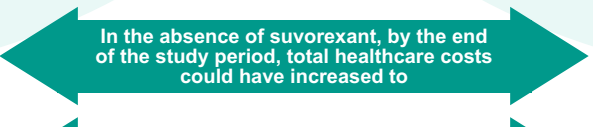

$\$ 878$

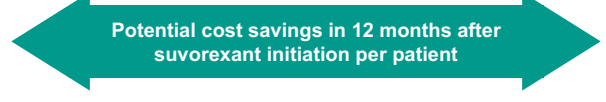

$\$ 1,405$

CHANGES IN TOTAL HEALTHCARE COSTS BEFORE AND AFTER SUVOREXANT ADMINISTRATION*

Prevalent Cohort Data Analysis

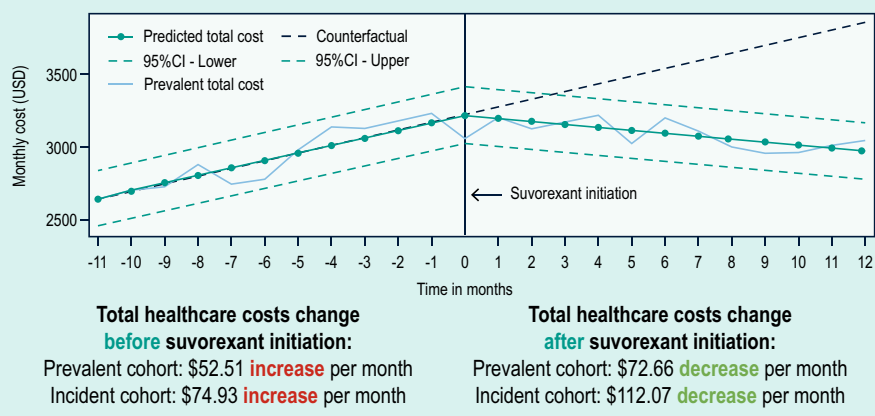

'Please refer to the journal article for a full analysis of prevalent and incident cohort data.

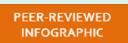

\section{Adis}

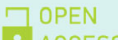

This graphical abstract represents a summary of the article. For a full list of declarations, including funding and author disclosure statements, please see the full text online. @The authors, CC-BY-NC 2021 
Keywords: Economic burden; Healthcare costs; Insomnia; Real-world evidence; Resource use; Suvorexant; Time series

\section{Key Summary Points}

Insomnia disorder is associated with poor quality of life and a significant clinical and economic burden in the United States.

The US Food and Drug Administration (FDA) approved suvorexant (Belsomra; Merck, Kenilworth, NJ, USA), an orexinreceptor antagonist, for the treatment of insomnia in August 2014.

Little is known about the effect of suvorexant on healthcare resource use (HCRU) and costs among insomnia patients in a real-world setting. Therefore, this study assessed the change in realworld HCRU and costs associated with suvorexant introduction among patients with insomnia in the US.

This study used an interrupted time series design with segmented regression to measure changes in trends for HCRU and costs in 12 months before and 12 months after suvorexant initiation.

The study found that suvorexant initiation was associated with immediate and continued decreases in all-cause HCRU and costs among patients with insomnia; larger reductions were observed in patients with mental health conditions.

Future studies would be needed to assess the causal link between suvorexant and economic outcomes by comparing patients on suvorexant to those receiving other treatments.

\section{DIGITAL FEATURES}

This article is published with digital features, including a graphical abstract, to facilitate understanding of the article. To view digital features for this article go to https://doi.org/10. 6084/m9.figshare.16884853.

\section{INTRODUCTION}

Insomnia disorder is associated with a significant burden in the United States (US) $[1,2]$. While the true prevalence of insomnia in the US is believed to range from 10 to $25 \%$, prevalence estimates have been reported to vary between 5 and $50 \%$ depending on the definition of insomnia or diagnostic criteria used [1, 3-5]. Insomnia is more common among women, older adults, and patients with physical or mental health disorders $[1,6]$. Untreated insomnia may result in an inability to focus, increased risk of accidents, reduced immune function, and the onset and/or worsening of psychiatric and medical comorbidities $[3,4]$. A diagnosis of insomnia has been associated with worse health-related quality of life compared with non-insomnia patients, and was found to have a substantial effect on loss of work productivity and employment-related outcomes $[2,7-9]$. The aggregate total cost of insomnia in the US has been estimated to be as high as US $\$ 100$ billion per year, driven primarily by indirect costs such as decreased work productivity, increased accident risk, and increased healthcare resource use (HCRU) [2]. In one study, patients with insomnia had $\$ 63,607$ higher medical costs than patients without insomnia; inpatient costs were the most significant driver of the direct medical cost [10].

While non-pharmacological treatments such as cognitive behavioral therapy are available and effective to treat insomnia, some patients may need to use pharmacotherapies such as benzodiazepine receptor agonists, melatonin agonists, antidepressants, antipsychotics, or antihistamines [6]. Benzodiazepine receptor agonists and benzodiazepines are among the most commonly used medications for insomnia, but there are concerns about the safety, risks of dependence and tolerance, and longterm effectiveness of these agents. Physicians also prescribe sedating antidepressants, antipsychotics, and antihistamines off-label to treat insomnia, despite insufficient efficacy 
evidence for these treatments and the risk of serious side effects, such as weight gain, anticholinergic effects, and diabetes $[6,11]$.

The US Food and Drug Administration (FDA) approved suvorexant (Belsomra; Merck, Kenilworth, NJ, USA), an orexin-receptor antagonist, for the treatment of insomnia in August 2014 [12]. Unlike benzodiazepines or benzodiazepine receptor agonists, which act on the gamma aminobutyric acid receptor, suvorexant specifically targets the orexin-mediated wakefulnesspromoting system by selectively blocking the binding of orexin-A and orexin-B neuropeptides to OX1R and OX2R receptors [13-16]. In addition, two systematic reviews and meta-analyses have found that suvorexant improved sleep quality in patients with insomnia $[17,18]$.

Little is known about the economic impact of treatment with hypnotics in patients with insomnia. Further, no studies to our knowledge have evaluated the economic impact of suvorexant in patients diagnosed with insomnia. Understanding the economic impact of suvorexant use among US patients with insomnia may provide important insight for payers and other healthcare decision-makers. The objective of this study was to evaluate the change in real-world HCRU and cost outcomes associated with suvorexant introduction among patients with insomnia in the US.

\section{METHODS}

\section{Data Source}

We conducted this retrospective cohort study using de-identified medical and pharmacy claims data from the US nationwide Optum Clinformatics Data Mart claims database (Optum, Eden Prairie, MN, USA). The database contains detailed information on insurance enrollment and coverage (commercial and Medicare); medical care (e.g., diagnosis codes, dates and place of service, provider type); prescription drug use [e.g., National Drug Code (NDC), drug quantity dispensed, days supplied]; reimbursed or allowed payments; deductibles; and copayments.
The Optum data received for the analysis was a secondary database, which included de-identified patient data. The secondary use of deidentified data is explicitly exempted from ethics review per the Department of Health and Human Services regulations found at 45 CFR 46.104(d)(4). Study authors received permission to use the Optum Clinformatics Data Mart claims database from Optum through a thirdparty agreement.

\section{Study Population}

The study population included adult patients (aged $\geq 18$ years) diagnosed with insomnia [International Classification of Diseases, Ninth Revision, Clinical Modification (ICD-9-CM) codes 780.5X, 327.XX, and 307.4X, or ICD-10CM codes F51.XX and G47.XX] from January 1, 2010, through December 31, 2018 (study period) who newly initiated suvorexant. All patients were required to have $\geq 1$ pharmacy claim for suvorexant during the study period. The index date was defined as the date of the first claim for suvorexant. A 12-month baseline period before the index date was used to identify patient demographic and clinical characteristics. The prevalent insomnia cohort included patients with newly and previously diagnosed insomnia (i.e., $\geq 2$ claims with an insomnia diagnosis during baseline period) and continuous insurance enrollment for $\geq 12$ months before and $\geq 6$ months after index date (Supplemental Fig. 1A). To identify patients who were new users of suvorexant, patients with any record of suvorexant use during the baseline period were excluded from the prevalent insomnia cohort. However, there were no limits regarding additional insomnia medication use after the baseline period.

A group of suvorexant users who were newly diagnosed with insomnia was also analyzed (incident insomnia cohort), and included patients with $\geq 2$ claims with an insomnia diagnosis and continuous enrollment from $\geq 12$ months prior to first insomnia diagnosis through $\geq 6$ months after index date (Supplemental Fig. 1B). Patients with any record of insomnia-specific drug use (suvorexant, 
flurazepam, temazepam, triazolam, estazolam, quazepam, zolpidem, zaleplon, eszopiclone, ramelteon, secobarbital, doxepin, diphenhydramine, doxylamine) or insomnia diagnosis in 12 months before the first observed insomnia diagnosis, or who were not continuously enrolled between insomnia diagnosis and suvorexant index date, were excluded from the incident insomnia cohort.

\section{Study Design}

We used an interrupted time series design and segmented regression analysis to assess the association of suvorexant initiation with HCRU and costs. In this method, a time series [i.e., a continuous sequence of observations on a population (aggregate) level taken at regularly spaced intervals over time] is used to establish an underlying level and trend of an outcome of interest, which is interrupted by an intervention at a known time point $[19,20]$. The preand post-intervention periods are then compared to estimate the impact of the intervention independent of other factors. Interrupted time series analysis is a strong quasi-experimental design that is increasingly being used to evaluate public health interventions, as it allows researchers to control for secular trends in the data, use population-level data to evaluate outcomes, and provides a clear graphical presentation of results [19-21].

\section{Study Outcomes}

Suvorexant initiation was the main independent variable and suvorexant use was identified using NDCs from prescription drug claims (Supplemental Table 1). The study outcomes were all-cause HCRU and associated costs, which were calculated at monthly intervals. Allcause HCRU included outpatient visits, officebased physician visits, inpatient hospitalizations, emergency department (ED) visits, other HCRU (i.e., home health, hospice care, and visits related to miscellaneous reasons in the database), and a number of prescription drugs. Rates of HCRU were converted to per 1000 patients to make interpretation easier than raw units. All-cause costs included an outpatient, office-based physician, inpatient, ED, and other costs; prescription drug costs; medical costs (sum of outpatient, office-based physician, inpatient, ED, and other costs); and total healthcare costs (sum of all cost types). Baseline demographic characteristics included age at suvorexant initiation, sex, insurance type (Medicare vs. commercial), and US Census region. Baseline clinical characteristics included Charlson comorbidity index (CCI) score and the following mental health comorbidities: depressive disorders, attention deficit hyperactivity disorder (ADHD), anxiety disorders, substance use disorder, and psychotic disorders (i.e., schizophrenia, bipolar disorder).

\section{Statistical Analysis}

Summary statistics (e.g., mean, median, standard deviation. and percentages) were used to describe patient demographic and clinical characteristics. Mean HCRU and costs per month were calculated for 12 months before the index date and 12 months after the index date. All costs were inflated to 2018 US dollars using the medical component of the Consumer Price Index.

A segmented regression approach was used to analyze an interrupted time series of monthly HCRU and costs for 12 months before and 12 months after suvorexant initiation [20]. The time series of outcomes data were divided into 2 segments: monthly rates before suvorexant initiation and monthly rates after suvorexant initiation. The regression model calculated changes in intercept (level) and slope (trend) in the post-intervention period compared to the pre-intervention period. The statistical model used was:

$$
\begin{aligned}
Y_{t}= & \beta_{0}+\beta_{1} * \text { time }_{t}+\beta_{2} * \text { intervention }_{t}+\beta_{3} \\
& * \text { time after intervention } \\
t & +e_{t}
\end{aligned}
$$

where $Y_{t}$ is the mean outcome (i.e., HCRU or cost) in month $t$, time is a continuous variable indicating time in months at time $t$ from the start of the observation period, intervention is an indicator for time $t$ occurring before 
(intervention $=0$ ) or after (intervention $=1$ ) suvorexant initiation, and time after intervention is a continuous variable counting the number of months after the intervention at time $t$. In this model, $\beta_{0}$ estimates the baseline level of the HCRU or cost at time zero, $\beta_{1}$ estimates the change in the mean HCRU or cost that occurs with each month before the intervention (i.e., the baseline trend), $\beta_{2}$ estimates the level change in the mean monthly HCRU or cost immediately after the intervention, that is, from the end of the preceding segment, and $\beta_{3}$ estimates the change in the trend in the mean monthly HCRU or cost after suvorexant initiation, compared with the monthly trend before suvorexant initiation. The sum of $\beta_{1}$ and $\beta_{3}$ is the post-intervention slope. The error term $e_{\mathrm{t}}$ at time $t$ represents the random variability not explained by model [20]. Predicted costs and HCRU were calculated from the regression models.

To understand trends in HCRU and cost outcomes in the absence of suvorexant, we calculated counterfactual scenarios: hypothetical scenarios in which suvorexant initiation did not occur and the pre-existing trend observed pre-suvorexant initiation was extrapolated, assuming a linear trend $[19,20]$.

Several patient-level factors (e.g., age, sex, comorbidities) can influence resource utilization and the relationship between suvorexant initiation and HCRU and costs. Therefore, as sensitivity analyses, we used patient-level data to conduct fixed-effect segmented regressions controlling for age at suvorexant initiation, sex, race/ethnicity, insurance type, US Census location, and CCI score as covariates and accounting for the repeated nature of longitudinal data.

Since insomnia is more prevalent among patients with mental health comorbidities compared with the general population [22], allcause costs for suvorexant patients with depressive disorders, anxiety disorder, substance use disorder, and psychotic disorders (Supplemental Table 2) were analyzed as separate subgroups of interest. All analyses were conducted separately among the prevalent insomnia cohort and the incident insomnia cohort using SAS v.9.4; SAS Institute, Cary, NC, USA).

\section{RESULTS}

\section{Patient Characteristics}

A total of 18,919 patients with prevalent insomnia who were new users of suvorexant were included in this analysis; the cohort of incident insomnia patients included 5939 patients (Fig. 1). Among all new suvorexant users (i.e., prevalent cohort), the mean patient age was 64.5 years, approximately $65 \%$ were female, $74 \%$ were covered by Medicare, $48 \%$ had a CCI score of 2 or more, $44 \%$ had an anxiety disorder, $41 \%$ had a depressive disorder, and $22 \%$ had substance use disorder at baseline. The incident insomnia cohort was relatively younger and had lower CCI scores and a lower prevalence of mental health comorbidities at baseline compared with the prevalent insomnia cohort (Table 1).

\section{Changes in HCRU After Suvorexant Initiation}

The monthly trends for all-cause HCRU before suvorexant initiation were increasing for outpatient, office-based physician, inpatient, and ED visits in both prevalent insomnia (Fig. 2) and incident insomnia cohorts (Fig. 3). After suvorexant initiation, the monthly rate of resource use decreased and the change in slopes between the pre- and post-suvorexant initiation periods were statistically significant in both cohorts (Table 2). In both prevalent and incident insomnia cohorts, outpatient and officebased visits per 1000 patients decreased immediately after initiation of suvorexant (level changes). Similarly, the trend in resource use (monthly change) decreased significantly after suvorexant initiation. The differences in the slopes between pre- and post-suvorexant initiation periods were statistically significant (outpatient visits: -24.38 in prevalent insomnia cohort, -28.69 in incident insomnia cohort, both $p<0.0001$; office-based physician visits: -40.08 in prevalent insomnia cohort, -46.20 in incident insomnia cohort, both $p<0.0001$ ). Prescription drug events increased after suvorexant initiation, as indicated by level 


\section{(A)}

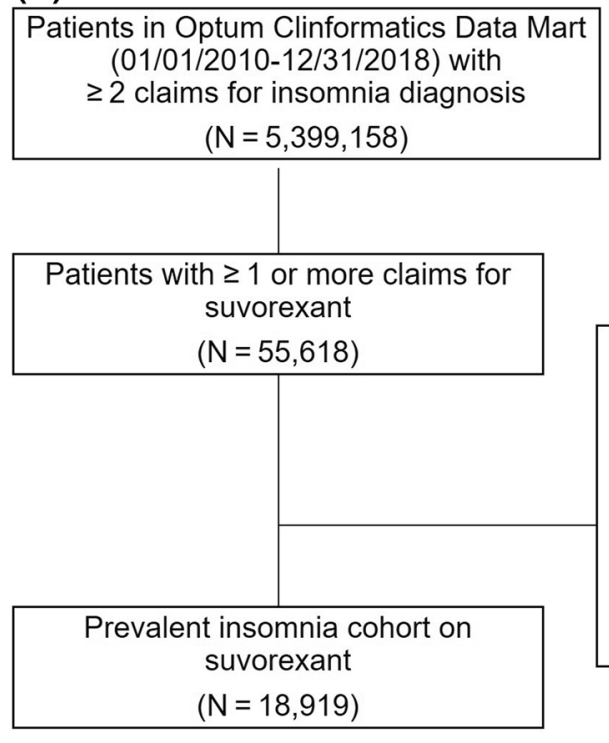

\section{Excluded:}

- Patients without $\geq 1$ claim for insomnia diagnosis during 12 months before suvorexant initiation $(n=24,591)$

- Patients without continuous health plan enrollment for 12 months before and 6 months after suvorexant initiation $(n=12,107)$

- Patients aged $<18$ years at insomnia diagnosis $(n=1)$

\section{(B)}

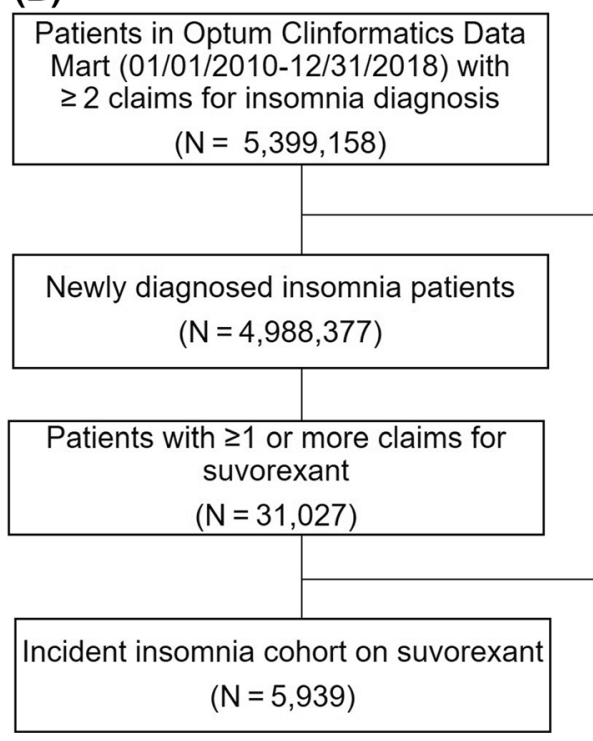

\section{Excluded:}

- Patients with prior insomnia diagnosis or insomnia medication use during 12-month baseline $(n=410,781)$

\section{Excluded: \\ - Patients without continuous health plan enrollment for 12 months before and 6 months after insomnia diagnosis $(n=25,088)$}

Fig. 1 Patient selection for a prevalent insomnia cohort and $\mathbf{b}$ incident insomnia cohort

change per 1000 patients (9.09 in prevalent insomnia cohort, $p=0.0706 ; 18.29$ in incident insomnia cohort, $p=0.0055)$, but the trend decreased significantly over time. The difference in the slopes between the pre- and post-suvorexant initiation periods was -5.29 in prevalent insomnia cohort and -7.39 in incident insomnia cohort (both $p<0.0001$; Table 2).

\section{Changes in Healthcare Costs After Suvorexant Initiation}

The trend for monthly all-cause total healthcare cost was increasing before suvorexant initiation (\$52.51 per month in prevalent insomnia cohort, $\$ 74.93$ per month in incident insomnia cohort, both $p<0.0001$; Table 3), but, after suvorexant initiation, the monthly total cost 
Table 1 Patient demographic and clinical characteristics

\begin{tabular}{|c|c|c|}
\hline & $\begin{array}{l}\text { Prevalent } \\
\text { insomnia } \\
\text { cohort }^{\mathrm{a}} \\
(n=18,919)\end{array}$ & $\begin{array}{l}\text { Incident } \\
\text { insomnia } \\
\text { cohort }^{b} \\
(n=5939)\end{array}$ \\
\hline $\begin{array}{l}\text { Age at suvorexant } \\
\text { initiation, mean }(\mathrm{SD}) \text {, } \\
\text { years }\end{array}$ & $64.5(14.1)$ & $62.8(15.5)$ \\
\hline \multicolumn{3}{|l|}{ Age category, years, $n(\%)$} \\
\hline $18-44$ & $1927(10.2)$ & $873(14.7)$ \\
\hline $45-54$ & $2483(13.1)$ & $796(13.4)$ \\
\hline $55-64$ & $3539(18.7)$ & $935(15.7)$ \\
\hline $65-74$ & $6264(33.1)$ & $1924(32.4)$ \\
\hline$\geq 75$ & $4698(24.8)$ & $1411(23.8)$ \\
\hline Female, $n(\%)$ & $12,252(64.8)$ & $3755(63.2)$ \\
\hline \multicolumn{3}{|c|}{ US Census location, $n(\%)$} \\
\hline Northeast & $1168(6.2)$ & $392(6.6)$ \\
\hline Midwest & $3650(19.3)$ & $1066(17.9)$ \\
\hline West & $4356(23.0)$ & $1580(26.6)$ \\
\hline South & $9643(51.0)$ & $2867(48.3)$ \\
\hline Unknown & $103(0.5)$ & $34(0.6)$ \\
\hline \multicolumn{3}{|l|}{ Insurance type, $n(\%)$} \\
\hline Medicare & $13,919(73.6)$ & $4213(70.9)$ \\
\hline Commercial & $5001(26.4)$ & $1726(29.1)$ \\
\hline \multicolumn{3}{|l|}{ Plan type, $n(\%)$} \\
\hline $\begin{array}{l}\text { Independent provider } \\
\text { organization/other }\end{array}$ & $9694(51.2)$ & $2961(49.9)$ \\
\hline $\mathrm{HMO} / \mathrm{POS}$ & $6625(35.0)$ & $2288(38.5)$ \\
\hline $\mathrm{PPO} / \mathrm{EPO}$ & $2601(13.7)$ & $690(11.6)$ \\
\hline CCI score, mean (SD) & $2.3(2.6)$ & $1.7(2.3)$ \\
\hline \multicolumn{3}{|l|}{ CCI category, $n(\%)$} \\
\hline 0 & $5973(31.6)$ & $2459(41.4)$ \\
\hline 1 & $3767(19.9)$ & $1156(19.5)$ \\
\hline 2 & $2657(14.0)$ & $799(13.5)$ \\
\hline$\geq 3$ & $6523(34.5)$ & $1525(25.7)$ \\
\hline
\end{tabular}

Table 1 continued

\begin{tabular}{lll}
\hline & $\begin{array}{l}\text { Prevalent } \\
\text { insomnia } \\
\text { cohort }^{\mathbf{a}} \\
(\boldsymbol{n}=\mathbf{1 8 , 9 1 9})\end{array}$ & $\begin{array}{l}\text { Incident } \\
\text { insomnia }^{\text {cohort }^{\mathbf{b}}} \\
(\boldsymbol{n}=\mathbf{5 9 3 9})\end{array}$ \\
\hline $\begin{array}{l}\text { Mental health comorbidities, } n(\%) \\
\begin{array}{l}\text { Depressive } \\
\text { disorders }\end{array}\end{array}$ & $7813(41.3)$ & $1618(27.2)$ \\
ADHD & $328(1.7)$ & $108(1.8)$ \\
Anxiety disorders & $8342(44.1)$ & $1579(26.6)$ \\
$\begin{array}{l}\text { Substance use } \\
\text { disorder }\end{array}$ & $4160(22.0)$ & $925(15.6)$ \\
$\begin{array}{l}\text { Psychotic } \\
\text { disorders }\end{array}$ & $1623(8.6)$ & $323(5.4)$
\end{tabular}

Insomnia medication before first observed insomnia diagnosis claim $^{\mathrm{d}}$

\begin{tabular}{lrr} 
Doxepin & $103(0.5)$ & NA \\
Estazolam & $21(0.1)$ & NA \\
Eszopiclone & $250(1.3)$ & NA \\
Flurazepam & $56(0.3)$ & NA \\
Quazepam & $2(0.0)$ & NA \\
Ramelteon & $694(3.7)$ & NA \\
Triazolam & $249(1.3)$ & NA \\
(Halcion) & & \\
Zaleplon & $367(1.9)$ & NA \\
Zolpidem & $7578(40.1)$ & NA \\
\hline
\end{tabular}

$A D H D$ attention deficit hyperactivity disorder, $C C I$ Charlson Comorbidity Index, EPO exclusive provider organization, HMO Health Maintenance Organization, $N A$ not applicable, PPO preferred provider organization, POS point of service, US United States

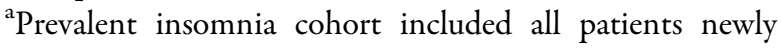
initiating suvorexant with newly diagnosed or previously diagnosed insomnia

${ }^{\mathrm{b}}$ Incident insomnia cohort included patients newly initiating suvorexant with newly diagnosed insomnia only ${ }^{c}$ Psychotic disorders included schizophrenia and bipolar disorders

${ }^{\mathrm{d}}$ Incident insomnia cohort excluded patients with any prior insomnia medications before first observed insomnia diagnosis 

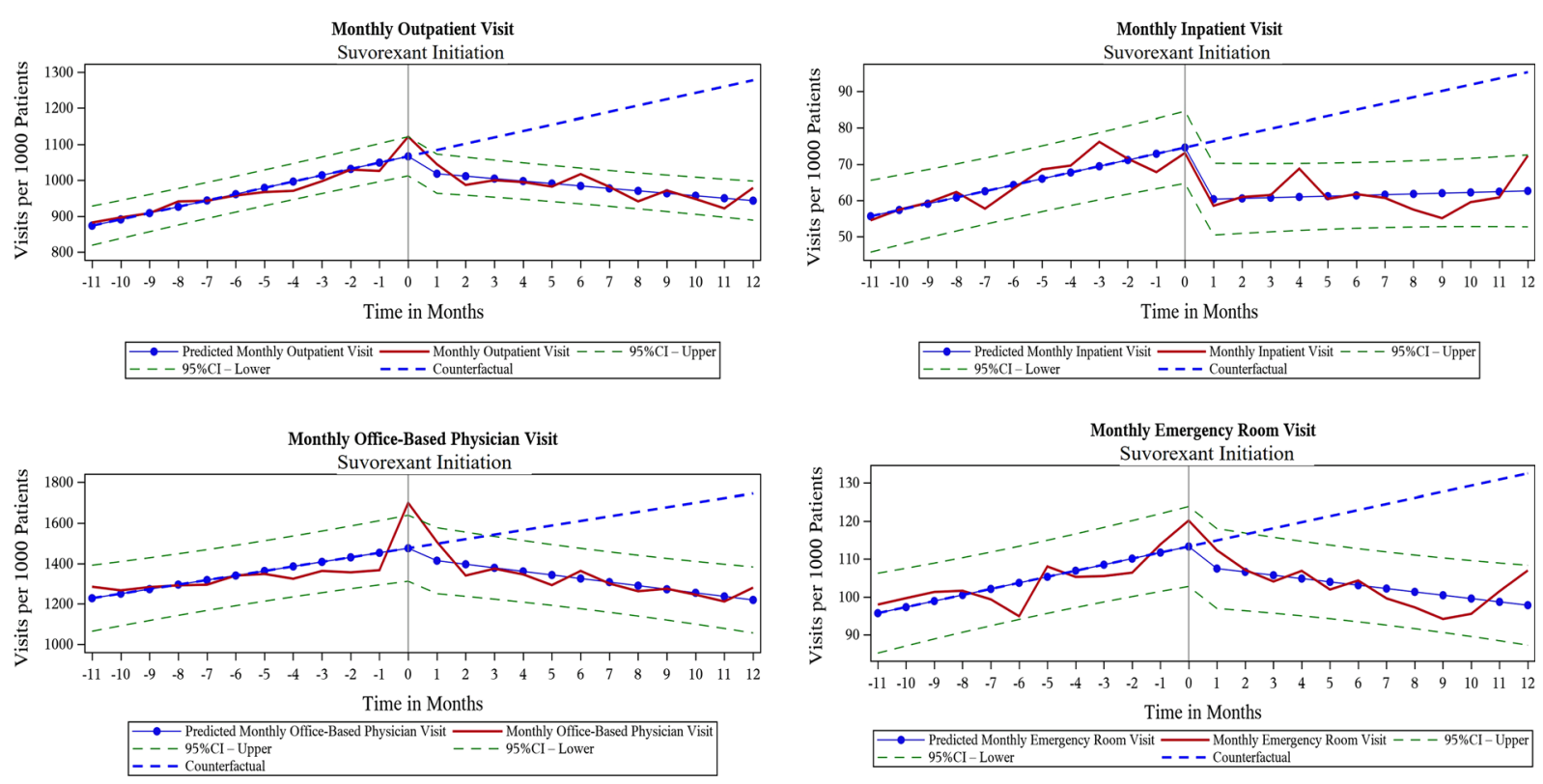

Fig. 2 Trends over time for monthly HCRU before and after initiation of suvorexant for prevalent insomnia cohort (HCRU healthcare resource use)
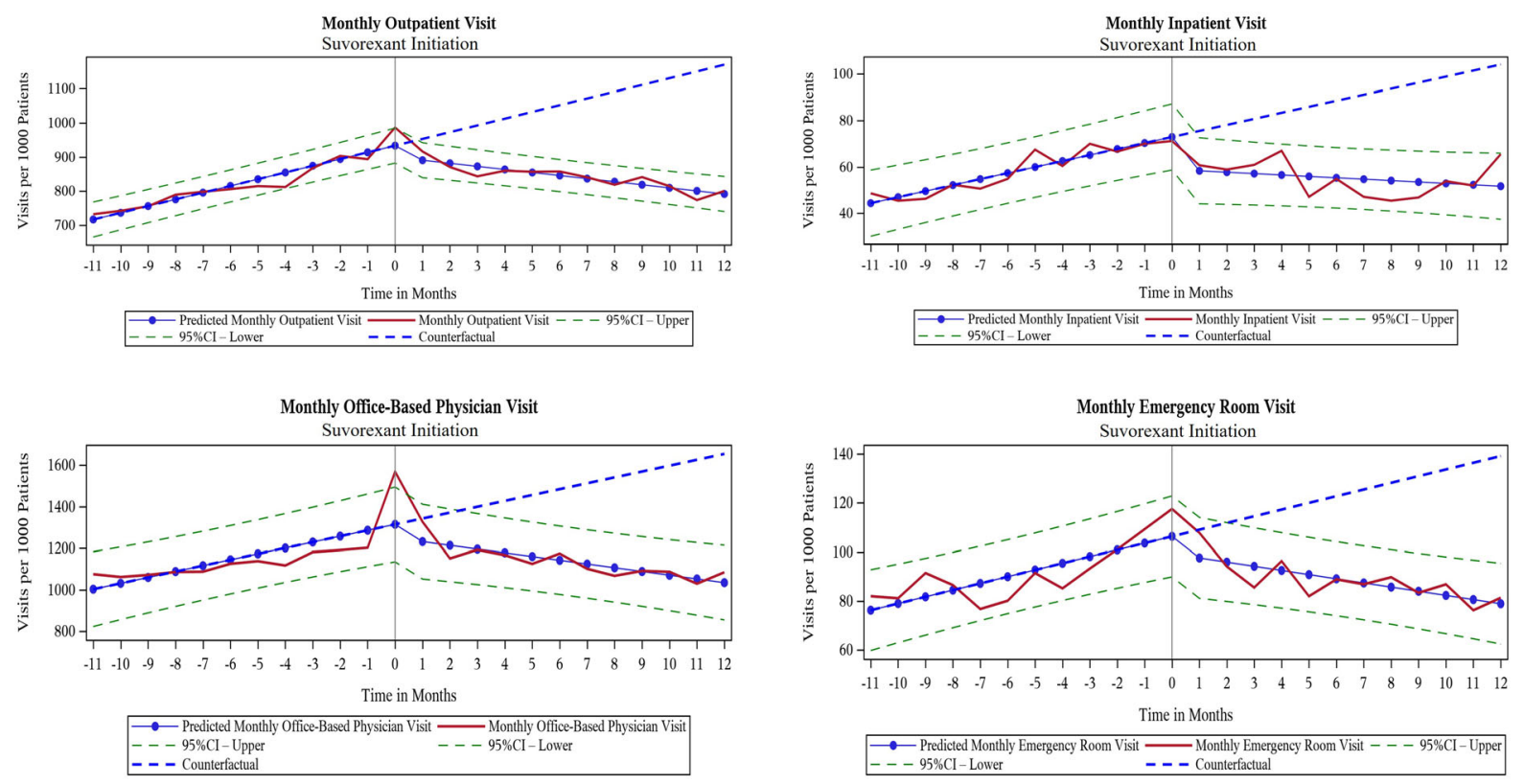

Fig. 3 Trends over time for monthly HCRU before and after initiation of suvorexant for incident insomnia cohort (HCRU healthcare resource use)

showed a decreasing trend in both cohorts (Fig. 4). The difference in the slope for total costs after suvorexant initiation was $\$ 72.66$ per month in the prevalent insomnia cohort and $\$ 112.07$ in the incident insomnia cohort, which was statistically significant (both $p<0.0001$; Table 3). This suggests that average cost per patient per year would decrease by $\$ 872$ and $\$ 1344$ in the prevalent and incident insomnia cohorts respectively. For total costs, the level 
Table 2 Results from segmented regression for monthly all-cause HCRU before and after initiation of suvorexant

\begin{tabular}{|c|c|c|c|c|}
\hline & \multicolumn{4}{|c|}{ Monthly HCRU per 1000 patients $^{a}$} \\
\hline & \multicolumn{2}{|c|}{ Prevalent insomnia cohort } & \multicolumn{2}{|c|}{ Incident insomnia cohort } \\
\hline & Estimate (SE) & $p$ value & Estimate (SE) & $p$ value \\
\hline \multicolumn{5}{|l|}{ Outpatient visits } \\
\hline Baseline (intercept) & $856.06(14.12)$ & $<0.0001$ & $697.32(13.30)$ & $<0.0001$ \\
\hline Slope before suvorexant initiation & $17.56(1.92)$ & $<0.0001$ & $19.69(1.81)$ & $<0.0001$ \\
\hline Level change after suvorexant initiation & $-41.61(18.84)$ & 0.0390 & $-33.83(17.74)$ & 0.0711 \\
\hline Change in slope after suvorexant initiation & $-24.38(2.71)$ & $<0.0001$ & $-28.69(2.56)$ & $<0.0001$ \\
\hline \multicolumn{5}{|l|}{ Office-based physician visits } \\
\hline Baseline (intercept) & $1206.14(42.27)$ & $<0.0001$ & $975.39(46.61)$ & $<0.0001$ \\
\hline Slope before suvorexant initiation & $22.44(5.74)$ & 0.0009 & $28.30(6.33)$ & 0.0002 \\
\hline Level change after suvorexant initiation & $-43.74(56.37)$ & 0.4468 & $-64.96(62.16)$ & 0.3085 \\
\hline Change in slope after suvorexant initiation & $-40.08(8.12)$ & $<0.0001$ & $-46.20(8.96)$ & $<0.0001$ \\
\hline \multicolumn{5}{|l|}{ Inpatient visits } \\
\hline Baseline (intercept) & $53.91(2.57)$ & $<0.0001$ & $41.82(3.70)$ & $<0.0001$ \\
\hline Slope before suvorexant initiation & $1.72(0.35)$ & $<0.0001$ & $2.60(0.50)$ & $<0.0001$ \\
\hline Level change after suvorexant Initiation & $-14.46(3.43)$ & 0.0004 & $-13.99(4.93)$ & 0.0102 \\
\hline Change in slope after suvorexant initiation & $-1.52(0.49)$ & 0.0060 & $-3.20(0.71)$ & 0.0002 \\
\hline \multicolumn{5}{|l|}{ Emergency department visits } \\
\hline Baseline (intercept) & $94.13(2.73)$ & $<0.0001$ & $73.56(4.27)$ & $<0.0001$ \\
\hline Slope before suvorexant initiation & $1.60(0.37)$ & 0.0003 & $2.73(0.58)$ & 0.0001 \\
\hline Level change after suvorexant initiation & $-4.94(3.64)$ & 0.1899 & $-7.05(5.70)$ & 0.2301 \\
\hline Change in slope after suvorexant initiation & $-2.47(0.52)$ & 0.0001 & $-4.43(0.82)$ & $<0.0001$ \\
\hline \multicolumn{5}{|l|}{ Other visits $^{\mathrm{b}}$} \\
\hline Baseline (intercept) & $379.95(8.03)$ & $<0.0001$ & $437.74(12.81)$ & $<0.0001$ \\
\hline Slope before suvorexant initiation & $9.51(1.09)$ & $<0.0001$ & $13.53(1.74)$ & $<0.0001$ \\
\hline Level change after suvorexant Initiation & $-9.51(10.71)$ & 0.3852 & $-10.71(17.09)$ & 0.5380 \\
\hline Change in slope after suvorexant initiation & $-12.65(1.54)$ & $<0.0001$ & $-20.80(2.46)$ & $<0.0001$ \\
\hline \multicolumn{5}{|l|}{ Prescription drug events } \\
\hline Baseline (intercept) & $980.40(3.57)$ & $<0.0001$ & $953.56(4.41)$ & $<0.0001$ \\
\hline Slope before suvorexant initiation & $2.76(0.48)$ & $<0.0001$ & $4.22(0.60)$ & $<0.0001$ \\
\hline Level change after suvorexant initiation & $9.09(4.76)$ & 0.0706 & $18.29(5.88)$ & 0.0055 \\
\hline
\end{tabular}


Table 2 continued

\begin{tabular}{|c|c|c|c|c|}
\hline & \multicolumn{4}{|c|}{ Monthly HCRU per 1000 patients $^{a}$} \\
\hline & \multicolumn{2}{|c|}{ Prevalent insomnia cohort } & \multicolumn{2}{|c|}{ Incident insomnia cohort } \\
\hline & Estimate (SE) & $p$ value & Estimate (SE) & $p$ value \\
\hline Change in slope after suvorexant initiation & $-5.29(0.69)$ & $<0.0001$ & $-7.39(0.85)$ & $<0.0001$ \\
\hline
\end{tabular}

$H C R U$ healthcare resource use

${ }^{a}$ Rate of resource use was converted to per 1000 patients to make interpretation easier than raw units

${ }^{\mathrm{b}}$ Other visits included home health, hospice care, and visits related to miscellaneous reasons in the database

change (intercept change) after suvorexant initiation was not statistically significant for either cohort because as medical costs decreased, prescription drug costs increased at the time of suvorexant initiation (Table 3 ). Similar to the HCRU analysis, suvorexant users from the incident insomnia cohort showed a larger decrease $(-\$ 112.07)$ in total healthcare costs per month compared to the prevalent insomnia cohort (-\$72.66) (Table 3).

For medical costs, both level and slope decreased significantly $(p<0.0001)$ after suvorexant initiation in both cohorts (Table 3 ). The difference in the slope for medical costs was $\$ 92.18$ per month and \$52.01 per month for the incident and prevalent cohorts, respectively, which suggests that the average medical cost per patient per year would decrease by $\$ 1106$ and \$624 in the incident and prevalent insomnia cohorts, respectively. Substantial changes were observed for outpatient, office-based, and inpatient costs in both cohorts (Table 3).

Similar results were observed in the fixed-effect sensitivity analysis for both cohorts, with increasing trends for monthly all-cause total, medical, and prescription drug costs before suvorexant initiation, but trends decreasing significantly $(p<0.0001)$ after suvorexant initiation (Supplemental Table 1). Likewise, the mental health subgroup analysis showed decreasing trends in total costs among patients with mental health comorbidities in prevalent insomnia (Supplemental Fig. 2) and incident insomnia (Supplemental Fig. 3) cohorts and larger decreases in total costs post-suvorexant initiation compared to the overall cohorts.
Slope changes observed for patients with mental health comorbidities were 1.5-3 times the changes observed for the overall cohorts. The largest decrease was observed for patients with substance use disorder (Supplemental Table 4).

\section{DISCUSSION}

In this large, retrospective claims database study, we examined changes over time in HCRU and costs in patients diagnosed with insomnia who initiated suvorexant treatment. To our knowledge, this is the first real-world study to examine economic outcomes in insomnia patients taking suvorexant in the US overall or after initiation of treatment with suvorexant. This study used a pre-post quasi-experimental design to examine temporal trends in HCRU and costs before and after initiation of suvorexant. Since it was important to examine changes over time associated with suvorexant, we conducted an interrupted time series analysis within the quasi-experimental study design framework. Overall, the results of our interrupted time series analysis showed decreasing trends in monthly all-cause HCRU and costs after suvorexant initiation in patients with prevalent or incident insomnia. The decreasing trend in total costs was primarily driven by reductions observed in outpatient visits, officebased visits, and inpatient visits after suvorexant initiation. Although prescription drug costs increased at suvorexant initiation (level change), this increase was offset by the decrease in medical costs. The increase in the 
Table 3 Results from segmented regression for monthly healthcare costs (2018 US dollars) before and after initiation of suvorexant

\begin{tabular}{|c|c|c|c|c|}
\hline & \multicolumn{2}{|c|}{ Prevalent insomnia cohort } & \multicolumn{2}{|c|}{ Incident insomnia cohort } \\
\hline & Estimate (SE) & $p$ value & Estimate (SE) & $p$ value \\
\hline \multicolumn{5}{|l|}{ Total costs } \\
\hline Baseline (intercept) & $2587.91(51.17)$ & $<0.0001$ & $2210.89(56.40)$ & $<0.0001$ \\
\hline Slope before suvorexant initiation & $52.51(6.95)$ & $<0.0001$ & $74.93(7.66)$ & $<0.0001$ \\
\hline Level change after suvorexant initiation & $-6.34(68.24)$ & 0.9269 & $-60.30(75.21)$ & 0.4321 \\
\hline Change in slope after suvorexant initiation & $-72.66(9.83)$ & $<0.0001$ & $-112.07(10.84)$ & $<0.0001$ \\
\hline \multicolumn{5}{|l|}{ Medical cost } \\
\hline Baseline (intercept) & $2035.85(53.94)$ & $<0.0001$ & $1765.24(56.22)$ & $<0.0001$ \\
\hline Slope before suvorexant initiation & $44.23(7.33)$ & $<0.0001$ & $66.73(7.64)$ & $<0.0001$ \\
\hline Level change after suvorexant initiation & $-187.86(71.93)$ & 0.0167 & $-229.87(74.97)$ & 0.0061 \\
\hline Change in slope after suvorexant initiation & $-52.01(10.36)$ & $<0.0001$ & $-92.18(10.80)$ & $<0.0001$ \\
\hline \multicolumn{5}{|l|}{ Outpatient cost } \\
\hline Baseline (intercept) & $810.30(18.01)$ & $<0.0001$ & $705.27(24.79)$ & $<0.0001$ \\
\hline Slope before suvorexant initiation & $13.10(2.45)$ & $<0.0001$ & $16.75(3.37)$ & $<0.0001$ \\
\hline Level change after suvorexant initiation & $-54.42(24.02)$ & 0.0348 & $-41.17(33.06)$ & 0.2274 \\
\hline Change in slope after suvorexant initiation & $-19.47(3.46)$ & $<0.0001$ & $-25.00(4.76)$ & $<0.0001$ \\
\hline \multicolumn{5}{|l|}{ Office-based physician cost } \\
\hline Baseline (intercept) & $282.11(6.79)$ & $<0.0001$ & $235.64(9.63)$ & $<0.0001$ \\
\hline Slope before suvorexant initiation & $6.82(0.92)$ & $<0.0001$ & $10.44(1.31)$ & $<0.0001$ \\
\hline Level change after suvorexant initiation & $-30.37(9.05)$ & 0.0032 & $-39.12(12.85)$ & 0.0064 \\
\hline Change in slope after suvorexant initiation & $-8.19(1.30)$ & $<0.0001$ & $-14.50(1.85)$ & $<0.0001$ \\
\hline \multicolumn{5}{|l|}{ Inpatient cost } \\
\hline Baseline (intercept) & $589.34(38.04)$ & $<0.0001$ & $485.46(36.98)$ & $<0.0001$ \\
\hline Slope before suvorexant initiation & $15.42(5.17)$ & 0.0073 & $27.62(5.02)$ & $<0.0001$ \\
\hline Level change after suvorexant initiation & $-85.07(50.73)$ & 0.1091 & $-160.51(49.32)$ & 0.0040 \\
\hline Change in slope after suvorexant initiation & $-13.66(7.31)$ & 0.0763 & $-32.53(7.11)$ & 0.0002 \\
\hline \multicolumn{5}{|l|}{ Emergency department cost } \\
\hline Baseline (intercept) & $152.33(6.16)$ & $<0.0001$ & $117.35(7.97)$ & $<0.0001$ \\
\hline Slope before suvorexant initiation & $2.58(0.84)$ & 0.0058 & $2.97(1.08)$ & 0.0124 \\
\hline Level change after suvorexant initiation & $-5.55(8.21)$ & 0.5071 & $3.65(10.62)$ & 0.7345 \\
\hline Change in slope after suvorexant initiation & $-5.00(1.18)$ & 0.0004 & $-7.30(1.53)$ & 0.0001 \\
\hline
\end{tabular}


Table 3 continued

\begin{tabular}{|c|c|c|c|c|}
\hline & \multicolumn{2}{|c|}{ Prevalent insomnia cohort } & \multicolumn{2}{|c|}{ Incident insomnia cohort } \\
\hline & Estimate (SE) & $p$ value & Estimate (SE) & $p$ value \\
\hline \multicolumn{5}{|l|}{ Other $\cos \mathrm{t}^{\mathrm{a}}$} \\
\hline Baseline (intercept) & $201.77(8.96)$ & $<0.0001$ & $221.53(15.28)$ & $<0.0001$ \\
\hline Slope before suvorexant initiation & $6.31(1.22)$ & $<0.0001$ & $8.95(2.08)$ & 0.0003 \\
\hline Level change after suvorexant initiation & $-12.46(11.95)$ & 0.3093 & $7.27(20.38)$ & 0.7248 \\
\hline Change in slope after suvorexant initiation & $-5.69(1.72)$ & 0.0035 & $-12.85(2.94)$ & 0.0003 \\
\hline \multicolumn{5}{|l|}{ Prescription drug cost } \\
\hline Baseline (intercept) & $552.07(22.29)$ & $<0.0001$ & $445.65(23.26)$ & $<0.0001$ \\
\hline Slope before suvorexant initiation & $8.28(3.03)$ & 0.0127 & $8.20(3.16)$ & 0.0173 \\
\hline Level change after suvorexant initiation & $181.53(29.72)$ & $<0.0001$ & $169.56(31.02)$ & $<0.0001$ \\
\hline Change in slope after suvorexant initiation & $-20.65(4.28)$ & 0.0001 & $-19.90(4.47)$ & 0.0002 \\
\hline
\end{tabular}

US United States

${ }^{a}$ Other costs included costs for home health, hospice care, and visits related to miscellaneous reasons in the database
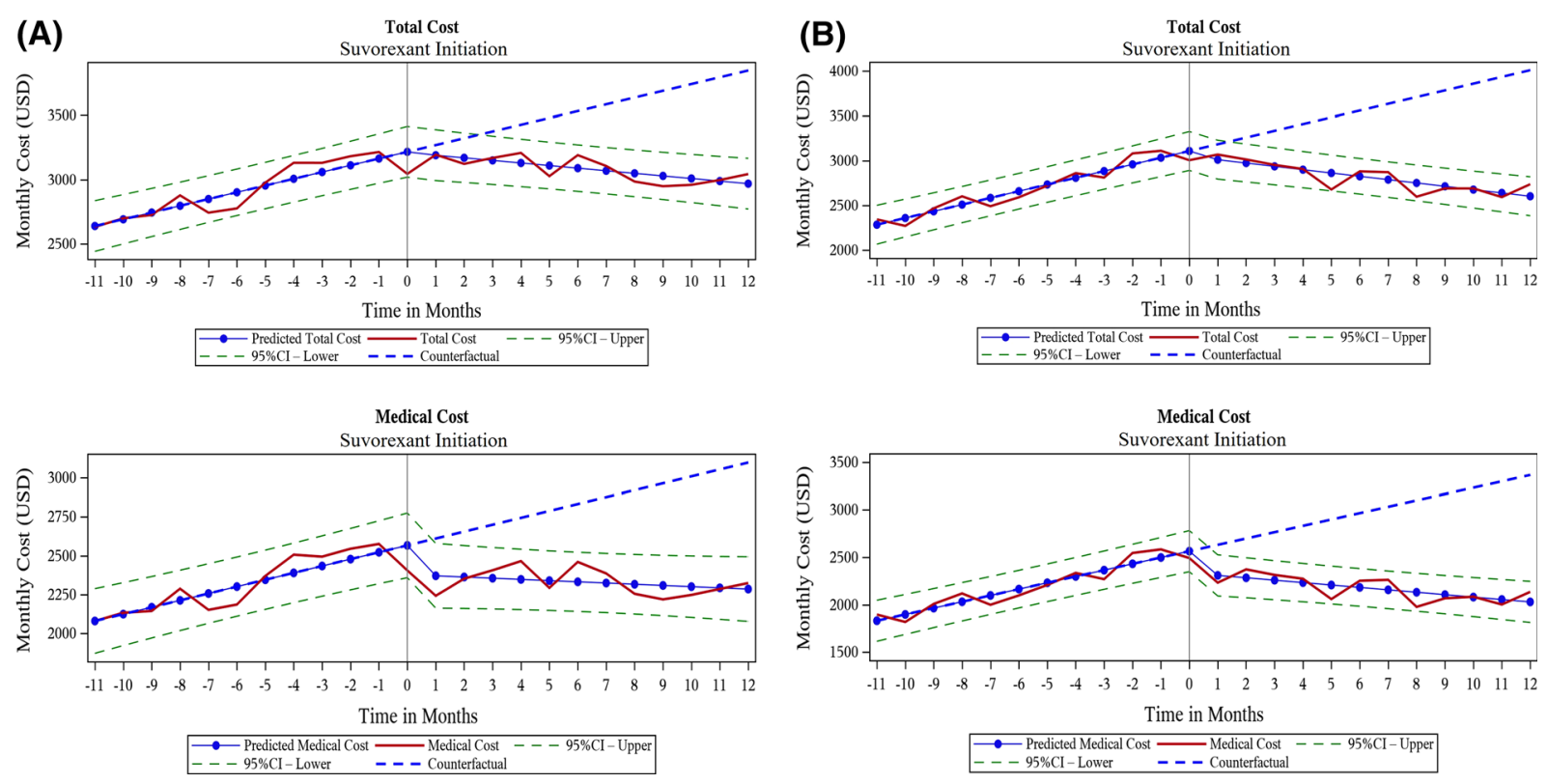

Fig. 4 Trend over time for monthly total healthcare costs and medical costs (2018 US dollars) before and after initiation of suvorexant for $\mathbf{a}$ prevalent insomnia cohort and $\mathbf{b}$ incident insomnia cohort. US United States

prescription costs at the time of suvorexant initiation could be due to suvorexant and other medications given to manage symptoms related to mental health or other comorbidities.
Further, slope changes suggested that prescription costs continued to decrease afterward.

While prior claims database analyses assessing real-world economic outcomes in patients with insomnia exist [23-26], none have yet 
focused specifically on patients treated with suvorexant. Therefore, it was not possible to compare our study findings with other studies. However, our findings are consistent with the conclusions derived from the cost-effectiveness analysis of suvorexant in the Japanese population, which reported that suvorexant was a costsaving strategy compared to zolpidem [27].

In a large claims database study that evaluated the use of several insomnia medications, direct medical cost reductions of $\$ 1100$ per patient per year were observed in patients who received pharmacotherapy for insomnia [28]. Burden of illness studies have found that untreated insomnia can result in significant healthcare costs with large proportions attributable to inpatient and outpatient costs [10]. In our study, we observed significant reductions in inpatient and outpatient utilization and associated costs after suvorexant initiation, suggesting that insomnia treatment with suvorexant may reduce healthcare costs.

Insomnia and mental health disorders have a bidirectional relationship [29, 30]. Research suggests that approximately $50 \%$ of chronic insomnia patients have mental health comorbidities, and that a majority of those with a mental health disorder have insomnia [22]. Further, untreated insomnia has been shown to worsen the underlying mental health conditions. Tian et al. found that patients with depression and insomnia had 1.5-2 times higher rates of resource use and annual costs than patients with depression alone [31]. Insomnia has also been linked to a higher chance of relapse in patients with substance use disorder [32, 33]. Therefore, we further examined the trends in HCRU and costs in patients with comorbid depression, anxiety disorder, substance use disorder, and psychotic disorders during the baseline period.

Our findings in the subgroup analyses suggested that decreases in all-cause cost slopes observed among patients with mental health disorders were nearly 1.5-3 times higher than the changes observed in the overall cohorts. The most notable decreases were observed in patients with substance use disorder, where the reduction in slope after suvorexant initiation was nearly \$305 per month for the incident insomnia cohort $(\sim 3$ times higher than the overall cohort). This suggests that suvorexant had a high impact on patients with mental health conditions, and that treating insomnia in patients with mental health disorders may result in clinical and economic benefits. Our findings are consistent with the Reducing Suicidal Ideation Through Insomnia Treatment (REST-IT) study, which found that insomnia treatment with zolpidem reduced the risk of suicidal ideation in patients with severe insomnia [34].

Currently, suvorexant is being studied in clinical trials in patients with substance use disorder and insomnia [35]. Our study findings from the subgroup of patients with substance use disorder suggest there are potential benefits associated with suvorexant.

This study has several strengths. As previously noted, it is the first study to evaluate HCRU and costs in patients with insomnia receiving suvorexant. The use of Optum data provided a large sample size and enabled analysis of newly diagnosed insomnia patients as well as prevalent insomnia patients who used suvorexant. Newly diagnosed patients had larger decreases in HCRU and costs than the prevalent insomnia cohort. This could be because newly diagnosed patients were relatively younger, had lower CCI scores, and had a lower prevalence of mental health conditions. Nevertheless, reductions in HCRU and costs were found for both cohorts. Next, the interrupted time series analysis design enabled us to control for secular trends in the outcomes data, and thereby reduce the potential bias of a simple pre- and post-intervention study design to provide more specific estimates of the impact of suvorexant initiation. We used a time series of monthly costs instead of fixed period costs, which allowed us to examine changes in both levels and trends of HCRU and costs. We also conducted sensitivity analyses using patientlevel data and fixed-effect regressions controlling for patient demographics and clinical characteristics, and accounting for the repeated nature of longitudinal data. Our findings from the patient-level models were consistent with the aggregate-level models. 
The study also has some limitations. It was a challenge to find a suitable control group due to heterogeneity present among patients with insomnia, as several disease conditions have a bidirectional relationship with insomnia. However, the quasi-experimental interrupted time series design allowed for patients to act as their own statistical "controls". We could not control for some covariates such as the severity of insomnia. Our analysis relied on the quality of claims data available in the Optum database, which may be subject to omissions, errors, or other differences in billing and reimbursement practices. There is an inherent risk of information bias resulting from data misclassification. In addition to suvorexant use, other factors may have contributed to reductions observed in costs. These may include patients receiving treatments for underlying disease conditions, such as cardiovascular disorders, diabetes, and mental health conditions. In our study, a large proportion of patients had mental health comorbidities, including depression, anxiety, and substance use disorder. It is possible that some of the reductions in the costs could be due to patients receiving treatment for mental health comorbidities in addition to suvorexant. We acknowledge that these treatments may have some confounding effects; however, patients with mental health conditions were present for the entire duration of the study, including the 12-months before and 12 months after suvorexant initiation. Additionally, in this study, we used a pre-post quasi-experimental design which assumes that patient-level confounders (observed and unobserved) are similar in the pre-and post-periods, which helps to minimize the confounding effect. Further, the study intended to only examine the association between suvorexant initiation and economic outcomes among patients initiating suvorexant. Therefore, causal inference between suvorexant initiation and economic outcomes cannot be ascertained. Future studies would be needed to assess the causal link between suvorexant treatment initiation and economic outcomes by comparing patients on suvorexant to those receiving other treatments. Additionally, future research can examine the effects of receiving suvorexant and concomitant medications on clinical and economic outcomes. In addition, the claims database did not include data on cognitive behavioral therapies for insomnia, the standard first-line treatment [6]. The study population was also limited to individuals with commercial and/or Medicare Advantage coverage captured in the Optum database, and therefore may not be representative of the entire US population. Further study findings are not generalizable to patients who were enrolled intermittently in their health plans, and who could be more vulnerable to the financial burden of treatments. While insomnia can have a significant impact on indirect costs (e.g., cost of lost productivity) and quality of life $[2,7,9]$, due to limitations of the Optum database, we could only assess direct medical costs in this analysis. Future studies can evaluate the effects of suvorexant initiation on indirect costs and quality of life outcomes.

\section{CONCLUSIONS}

In this real-world study, suvorexant initiation was associated with immediate and continued decreases within 1 year in all-cause HCRU and costs among patients with insomnia. Large cost decreases were observed for patients with comorbid mental health disorders. Further research is needed to understand the effect of suvorexant initiation on direct medical costs as well as costs associated with lost productivity in other settings.

\section{ACKNOWLEDGEMENTS}

Funding. This study and its submission for publication, including the journal's Rapid Service and Open Access Fees were funded by Merck Sharp \& Dohme Corp., a subsidiary of Merck \& Co., Inc., Kenilworth, NJ, USA.

Medical Writing Assistance. Medical writing support was provided by Catherine Mirvis (OPEN Health, Bethesda, MD) and funded by the study sponsor. 
Authorship. All named authors meet the International Committee of Medical Journal Editors (ICMJE) criteria for authorship for this article, take responsibility for the integrity of the work as a whole, and have given their approval for this version to be published.

Authors' Contributions. All authors contributed to the conceptualization and designing of the study, and drafting of the manuscript. Hrishikesh Kale, Ruchit Shah, Zaina Qureshi and Rezaul Khandker contributed to statistical analyses.

Disclosures. Hrishikesh Kale, Ruchit Shah and Marc Botteman are employees of OPEN Health, Bethesda, MD, USA and have nothing to disclose. Zaina Qureshi, Rezaul Khandker, Weilin Meng are employed by Merck Sharp \& Dohme Corp., a subsidiary of Merck \& Co., Inc., Kenilworth, NJ, USA and have nothing to disclose. At the time of study, Ruth Benca (RB) was an employee of University of California, Irvine, USA. RB also served as a consultant for Eisai, Genomind, Idorsia, Jazz, Merck, Sage, Sunovion and received a grant from Esai. RB is now an employee of Wake Forest Baptist Medical Center, North Carolina, USA.

Compliance with Ethics Guidelines. The data for this study was obtained from Optum Clinformatics database, which is an encrypted de-identified patient data. The secondary use of de-identified data is explicitly exempted from ethics review per the Department of Health and Human Services regulations found at 45 CFR 46.104(d)(4). Study authors received permission to use the Optum Clinformatics Data Mart claims database from Optum through a thirdparty agreement.

Data Availability. The data that support the study are available within the article and supplementary material.

Open Access. This article is licensed under a Creative Commons Attribution-NonCommercial 4.0 International License, which permits any non-commercial use, sharing, adaptation, distribution and reproduction in any medium or format, as long as you give appropriate credit to the original author(s) and the source, provide a link to the Creative Commons licence, and indicate if changes were made. The images or other third party material in this article are included in the article's Creative Commons licence, unless indicated otherwise in a credit line to the material. If material is not included in the article's Creative Commons licence and your intended use is not permitted by statutory regulation or exceeds the permitted use, you will need to obtain permission directly from the copyright holder. To view a copy of this licence, visit http://creativecommons.org/licenses/by$\mathrm{nc} / 4.0 /$.

\section{REFERENCES}

1. Morin CM, Jarrin DC. Epidemiology of insomnia: prevalence, course, risk factors, and public health burden. Sleep Med Clin. 2013;8(3):281-97. https:// doi.org/10.1016/j.jsmc.2013.05.002.

2. Wickwire EM, Shaya FT, Scharf SM. Health economics of insomnia treatments: the return on investment for a good night's sleep. Sleep Med Rev. 2016;30:72-82. https://doi.org/10.1016/j.smrv. 2015.11.004.

3. Ford ES, Cunningham TJ, Giles WH, Croft JB. Trends in insomnia and excessive daytime sleepiness among US adults from 2002 to 2012. Sleep Med. 2015;16(3):372-8. https://doi.org/10.1016/j. sleep.2014.12.008.

4. Klingman KJ, Sprey J. Insomnia disorder diagnosis and treatment patterns in primary care: a crosssectional analysis of electronic medical records data. J Am Assoc Nurse Pract. 2020;32(2):145-51. https://doi.org/10.1097/jxx.0000000000000232.

5. Roth T, Coulouvrat C, Hajak G, et al. Prevalence and perceived health associated with insomnia based on DSM-IV-TR; International Statistical Classification of Diseases and Related Health Problems, tenth revision; and research diagnostic criteria/international classification of sleep disorders, second edition criteria: results from the America Insomnia Survey. Biol Psychiatry. 2011;69(6): 592-600. https://doi.org/10.1016/j.biopsych.2010. 10.023 .

6. Sateia MJ, Buysse DJ, Krystal AD, Neubauer DN, Heald JL. Clinical practice guideline for the pharmacologic treatment of chronic insomnia in adults: 
an American Academy of Sleep Medicine clinical practice guideline. J Clin Sleep Med. 2017;13(2): 307-49. https://doi.org/10.5664/jcsm.6470.

7. Bolge SC, Joish VN, Balkrishnan R, Kannan H, Drake CL. Burden of chronic sleep maintenance insomnia characterized by nighttime awakenings. Popul Health Manag. 2010;13(1):15-20. https://doi. org/10.1089/pop.2009.0028.

8. Gu NY, Botteman MF, Ji X, Bell CF, Carter JA, van Hout B. Mapping of the Insomnia Severity Index and other sleep measures to EuroQol EQ-5D health state utilities. Health Qual Life Outcomes. 2011;9(1):119. https://doi.org/10.1186/1477-75259-119.

9. Ishak WW, Bagot K, Thomas S, et al. Quality of life in patients suffering from insomnia. Innov Clin Neurosci. 2012;9(10):13-26.

10. Wickwire EM, Tom SE, Scharf SM, Vadlamani A, Bulatao IG, Albrecht JS. Untreated insomnia increases all-cause health care utilization and costs among Medicare beneficiaries. Sleep. 2019;42(4): zsz007. https://doi.org/10.1093/sleep/zsz007.

11. Cunnington D, Junge MF, Fernando AT. Insomnia: prevalence, consequences and effective treatment. Med J Aust. 2013;199(S8):S36-40. https://doi.org/ 10.5694/mja13.10718.

12. US Food and Drug Administration, Center for Drug Evaluation and Research. Belsomra NDA 204569 approval letter, August 13, 2014. https://www. accessdata.fda.gov/drugsatfda_docs/appletter/2014/ 204569Orig1s000rltr.pdf. Accessed 7 May 2021.

13. BELSOMRA® (suvorexant) prescribing information. Merck Sharp \& Dohme Corp., Whitehouse Station, NJ, USA. Updated 03/2020. https://www.accessdata. fda.gov/drugsatfda_docs/label/2020/204569Orig1s 007lbl.pdf. Accessed 10 Mar 2021.

14. Seol J, Fujii Y, Park I, et al. Distinct effects of orexin receptor antagonist and $\mathrm{GABA}_{\mathrm{A}}$ agonist on sleep and physical/cognitive functions after forced awakening. Proc Natl Acad Sci USA. 2019;116(48):24353-8. https://doi.org/10.1073/pnas.1907354116.

15. Herring WJ, Connor KM, Ivgy-May $\mathrm{N}$, et al. Suvorexant in patients with insomnia: results from two 3-month randomized controlled clinical trials. Biol Psychiatry. 2016;79(2):136-48. https://doi.org/ 10.1016/j.biopsych.2014.10.003.

16. Michelson D, Snyder E, Paradis E, et al. Safety and efficacy of suvorexant during 1-year treatment of insomnia with subsequent abrupt treatment discontinuation: a phase 3 randomised, double-blind, placebo-controlled trial. Lancet Neurol. 2014;13(5):
$461-71$.

4422(14)70053-5.

https://doi.org/10.1016/S1474-

17. Kishi T, Matsunaga S, Iwata N. Suvorexant for primary insomnia: a systematic review and metaanalysis of randomized placebo-controlled trials. PLoS ONE. 2015;10(8): e0136910. https://doi.org/ 10.1371/journal.pone.0136910.

18. Kuriyama A, Tabata H. Suvorexant for the treatment of primary insomnia: a systematic review and meta-analysis. Sleep Med Rev. 2017;35:1-7. https:// doi.org/10.1016/j.smrv.2016.09.004.

19. Bernal JL, Cummins S, Gasparrini A. Interrupted time series regression for the evaluation of public health interventions: a tutorial. Int J Epidemiol. 2016;46(1):348-55. https://doi.org/10.1093/ije/ dyw098.

20. Wagner AK, Soumerai SB, Zhang F, Ross-Degnan D. Segmented regression analysis of interrupted time series studies in medication use research. J Clin Pharm Ther. 2002;27(4):299-309. https://doi.org/ 10.1046/j.1365-2710.2002.00430.x.

21. Penfold RB, Zhang F. Use of interrupted time series analysis in evaluating health care quality improvements. Acad Pediatr. 2013;13(6 Suppl):S38-44. https://doi.org/10.1016/j.acap.2013.08.002.

22. Soehner AM, Harvey AG. Prevalence and functional consequences of severe insomnia symptoms in mood and anxiety disorders: results from a nationally representative sample. Sleep. 2012;35(10):1367-75. https://doi.org/10.5665/sleep.2116.

23. Anderson LH, Whitebird RR, Schultz J, McEvoy CE, Kreitzer MJ, Gross CR. Healthcare utilization and costs in persons with insomnia in a managed care population. Am J Manag Care. 2014;20(5):e157-65.

24. Ozminkowski RJ, Wang S, Walsh JK. The direct and indirect costs of untreated insomnia in adults in the United States. Sleep. 2007;30(3):263-73. https:// doi.org/10.1093/sleep/30.3.263.

25. Pollack M, Seal B, Joish VN, Cziraky MJ. Insomniarelated comorbidities and economic costs among a commercially insured population in the United States. Curr Med Res Opin. 2009;25(8):1901-11. https://doi.org/10.1185/03007990903035505.

26. Wickwire EM, Vadlamani A, Tom SE, Johnson AM, Scharf SM, Albrecht JS. Economic aspects of insomnia medication treatment among Medicare beneficiaries. Sleep. 2020;43(1):zsz192. https://doi. org/10.1093/sleep/zsz192.

27. Nishimura S, Nakao M. Cost-effectiveness analysis of suvorexant for the treatment of Japanese elderly patients with chronic insomnia in a virtual cohort. 
J Med Econ. 2018;21(7):698-703. https://doi.org/ 10.1080/13696998.2018.1466710.

28. Jhaveri M, Seal B, Pollack M, Wertz D. Will insomnia treatments produce overall cost savings to commercial managed-care plans? A predictive analysis in the United States. Curr Med Res Opin. 2007;23(6):1431-43. https://doi.org/10.1185/ $030079907 X 199619$.

29. Jansson-Fröjmark M, Lindblom K. A bidirectional relationship between anxiety and depression, and insomnia? A prospective study in the general population. J Psychosom Res. 2008;64(4):443-9. https://doi.org/10.1016/j.jpsychores.2007.10.016.

30. Sivertsen B, Salo P, Mykletun A, et al. The bidirectional association between depression and insomnia: the HUNT study. Psychosom Med. 2012;74(7): 758-65. 0b013e3182648619. https://doi.org/10.1097/PSY.

31. Tian H, Abouzaid S, Gabriel S, Kahler KH, Kim E. Resource utilization and costs associated with insomnia treatment in patients with major depressive disorder. Prim Care Companion CNS Disord.
2012;14(5):PCC.12m01374. https://doi.org/10. 4088/PCC.12m01374.

32. Brower KJ, Perron BE. Sleep disturbance as a universal risk factor for relapse in addictions to psychoactive substances. Med Hypotheses. 2010;74(5): 928-33. https://doi.org/10.1016/j.mehy.2009.10. 020.

33. Roehrs TA, Roth T. Sleep disturbance in substance use disorders. Psychiatr Clin N Am. 2015;38(4): 793-803. https://doi.org/10.1016/j.psc.2015.07. 008.

34. McCall WV, Benca RM, Rosenquist PB, et al. Reducing Suicidal Ideation Through Insomnia Treatment (REST-IT): a randomized clinical trial. Am J Psychiatry. 2019;176(11):957-65. https://doi. org/10.1176/appi.ajp.2019.19030267.

35. The efficacy of suvorexant in treatment of patients with substance use disorder and insomnia: a pilot open trial (Suvsubuse). ClinicalTrials.gov identifier: NCT03412591. Updated July 13, 2020. Accessed April 7, 2021. https://clinicaltrials.gov/ct2/show/ NCT03412591 